\section{Case report: cobblestone}

\title{
Chlamydia trachomatis infection mimicking testicular malignancy in a young man
}

\author{
A M Ward, J H Rogers, C S Estcourt
}

A young man with a low risk history for sexually transmitted diseases presented with an apparently longstanding, previously asymptomatic scrotal mass, highly suggestive of testicular malignancy on palpation. Ultrasound sited the lesion in the epididymis. Although there was no evidence of urethritis, chlamydia polymerase chain reaction testing was positive. Tumour markers were negative. Complete clinical and radiological response was achieved after a long course of doxycycline treatment, without surgical exploration of the scrotum, confirming the diagnosis of chlamydial epididymitis.

(Sex Transm Inf 1999;75:270)

Keywords: testicular malignancy; Chlamydia trachomatis; epididymitis

A 36 year old Chinese man presented with a 2 day history of a sore scrotal lump. He had no urethral discharge or dysuria, and no history of sexually transmitted diseases. He denied any extramarital sexual partners since his marriage 5 years ago, but acknowledged four or five female partners before that. The couple had one child and were using condoms for contraception.

Examination revealed left sided scrotal swelling and a mildly tender mass, inseparable from the lower pole of the left testis, with an irregular surface and rock hard consistency. It did not transilluminate. There was no meatal discharge and no polymorphs on Gram stain of a urethral swab. An STD screen was performed. Trimethoprim was prescribed for a provisional diagnosis of non-sexually transmitted epididymitis secondary to a testicular tumour.

Urgent scrotal ultrasound revealed a $23 \mathrm{~mm}$ mass with a ragged margin and increased vascularity, located in the tail of the left epididymis. Urgent urological review was sought to obtain a histological diagnosis.

Urine chlamydia polymerase chain reaction (PCR) was positive. Urethral culture for gonorrhoea, a midstream urine sample, and tumour markers were all negative. The patient again denied other sexual partners in the past 5 years. Doxycycline was added.

After 1 week the lump was softer, less tender, and clearly palpable posterior to the left testis. The urologist felt the presentation was consistent with resolving epididymitis. Surgical exploration was deferred.

The patient required $21 / 2$ months of doxycycline treatment. Serial ultrasounds showed steady decrease in size of the mass until it was impalpable. His wife had a negative cervical chlamydia PCR but was treated with azithromycin as a contact.
Fifteen months later the patient was asymptomatic with normal examination and ultrasonography, and negative urinary chlamydia PCR. He declined semen analysis.

\section{Discussion}

Longstanding, subacute epididymitis, presenting with a painless scrotal mass, and without evidence of urethritis, is an unusual complication of chlamydial infection. ${ }^{1}$

Two cases of orchidectomy for presumptive cancer, in which the final diagnosis was epididymitis, have been reported..$^{2}$ One report described a case of chlamydial epididymitis in a 29 year old man, diagnosed by PCR on testicular tissue, after unilateral orchidectomy for a scrotal mass inseparable from the testis at surgical exploration. Contralateral epididymitis ensued 2 weeks later, responding to doxycycline treatment.

Cases such as these indicate it is preferable not to operate on a non-acute scrotal mass until results, including an STD screen, are available. This is particularly relevant in young men in whom sexually transmitted causes of epididymitis are common, and testicular malignancies are most prevalent. Had the chlamydia PCR result not been available quickly, this patient may have undergone an unnecessary surgical procedure for suspected malignancy.

1 Hori S, Tsutsumi Y. Histological differentiation between chlamydial and bacterial epididymitis: nondestructive and proliferative versus destructive and abscess formingimmunohistochemical and clinicopathological findings. Human Pathol 1995;26:402-7.

2 Slanetz PA, Whitman GJ, Chew FS. Epididymal abscess. Radiologic-Pathologic Conferences of the Massachusetts General Hospital. Am f Roentgenol 1995;164:376.

3 Molijn GJ, Bogdanowicz JF. Chlamydial epididymitis presenting as a solid asymptomatic scrotal mass. Br F Urol 1997;80:354. 